\title{
QUARKONIUM SPECTROSCOPY AND NEW STATES FROM BABAR
}

\author{
L. VITALE \\ Università and INFN Trieste, \\ Padriciano 99, \\ 34012, Trieste, ITALY \\ E-mail: lorenzo.vitale@ts.infn.it
}

\begin{abstract}
We review results on charmonium and bottomonium spectroscopy by the BaBar experiment at the PEP-II $e^{+} e^{-}$collider at SLAC. More space is reserved to the new results like the the observation of hadronic non- $B \bar{B} \Upsilon(4 S)$ decays and the investigation on the production and decay properties of the recently discovered charmonium-like states $X(3872)$ and $Y(4260)$. These results are preliminary, unless otherwise specified.
\end{abstract}

\section{Introduction}

Hadron spectroscopy plays an important role in validating the quark model and the quantum chromodinamics (QCD). In the recent years this field has seen a renewed interest, thanks to the discovery of many new states by the $B$ factories, BaBar at SLAC and Belle at KEK. In this contribution we concentrate on recent $\mathrm{BaBar}$ results in the heavy quarkonium spectroscopy both in bottom and charm quark sectors.

The BaBar experiment is taking data at or just below the $\Upsilon(4 S)$ resonance at the PEP-II asymmetric energy $e^{+} e^{-}$collider. BaBar and PEP-II were optimized for $C P$ violations studies in $B$ physics. Nevertheless, with the high integrated luminosity collected (about $330 \mathrm{fb}^{-1}$ until April 2006) and with the excellent performances of the detectors very good opportunities are available also in other fields, like the ones discussed here.

This contribution is organized as follows: in Sec. 2, for bottomonium, we report the studies of hadronic non- $B \bar{B} \Upsilon(4 S)$ decays to its lower radially excited states; in Sec. 3 we report the investigation of the properties of two recently observed puzzling states, $X(3872)$ and $Y(4260)$, that can be associated with charmonium (at least in their decays). 


\section{Bottomonium Results: hadronic non- $B \bar{B} \Upsilon(4 S)$ decays}

The $\Upsilon(4 S)$ resonance decays mostly to $B \bar{B}$, but not only. Branching fractions of the order $\mathcal{O}\left(10^{-4}\right)$ are expected for decays to $\pi^{+} \pi^{-} \Upsilon(n S)$, with $n=1,2(n=3$ is not kinematically allowed). Last year at LP Belle reported a first evidence for the decay $n=1$ that is still preliminary ${ }^{1}$.

BaBar has made a more optimized study based on $211 \mathrm{fb}^{-1}$ on peak, corresponding to $230 \cdot 10^{6} \Upsilon(4 S)$ decays, searching for decays to $\pi^{+} \pi^{-}$ $\Upsilon(n S)$, with $n=1,2$, that has been published just after this conference ${ }^{2}$. We observe a signal with a significance of 10. $\sigma$ in $n=1$ channel and a $7.3 \sigma$ signal in $n=2$ as seen in Fig.1. In particular this is the first evidence of the $\Upsilon(4 S) \rightarrow \pi^{+} \pi^{-} \Upsilon(2 S)$ decay. Partial widths and dipion
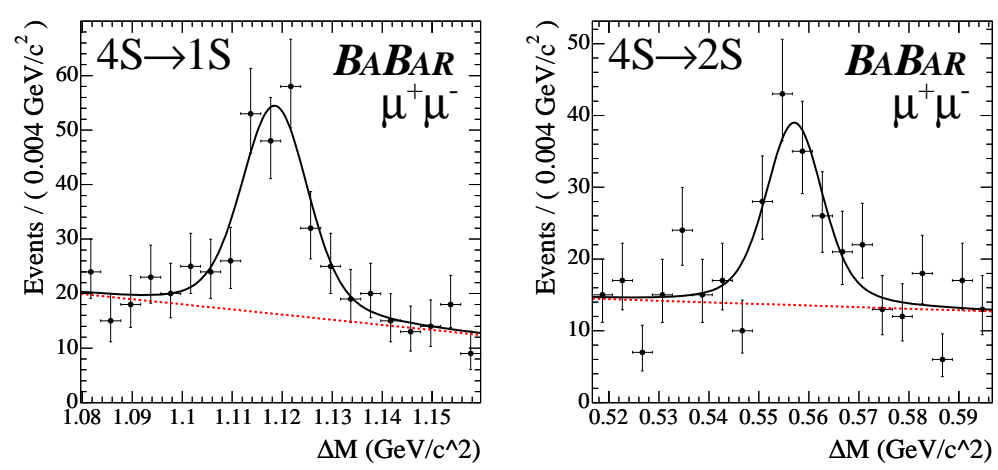

Figure 1. $\Delta M \Upsilon(4 S) \rightarrow \pi^{+} \pi^{-} \Upsilon(2 S)$ decay.

spectra are then compared with with QCD multipole expansion ${ }^{3}$ available for $\Upsilon(n S) \rightarrow \pi^{+} \pi^{-} \Upsilon(n S), m S \rightarrow n S$. We observe that the dipion spectra are compatible with predictions for $4 S \rightarrow 1 S$, but are not for $4 S \rightarrow 2 S$, confirming a discrepancy already seen in $3 S \rightarrow 1 S$.

\section{Charmonium Results}

Charmonium spectroscopy is a rich spectroscopy with properties that are well understood up to $\psi(3770)$, which is just above the threshold for open flavour production. In principle above this threshold, states are expected not to be narrow, and to decay dominantly to open charm channels ${ }^{4}$. 


\subsection{Studies of the narrow X(3872) state}

This good agreement between experimental data and theoretical predictions became more critical in 2003 with the discovery of a state close to $D^{0} D^{* 0}$ mass by BELLE ${ }^{5}$. A narrow peak in $J / \psi \pi^{+} \pi^{-}$mass shown up in $B^{-} \rightarrow J / \psi \pi^{+} \pi^{-} K^{-}$decays. This state, called $X(3872)$ was then confirmed by CDF, D0 and BaBar, all in the same decay mode ${ }^{6}$. Several possible interpretations have been formulated alternative to the ordinary $c \bar{c}$ charmonium state $\left(D^{0} D^{* 0}\right.$ molecule, tetraquark state, hybrid $c \bar{c} g$, vector glueball, etc.) and several experimental studies have been performed to discriminate among these hypothesis. The favored assignment of quantum numbers is $J^{P C}=1^{++}$.

We present the new published BaBar results with increased statistics ${ }^{7}$ in $B^{0} \rightarrow J / \psi \pi^{+} \pi^{-} K^{0}$ and $B^{-} \rightarrow J / \psi \pi^{+} \pi^{-} K^{-}$decays, were we find respectively a $2.5 \sigma$ and $6.1 \sigma$ signal (Fig.2). The ratio of branching fractions can already exclude some molecule model, while the difference in mass doesn't still exclude a tetraquark model.
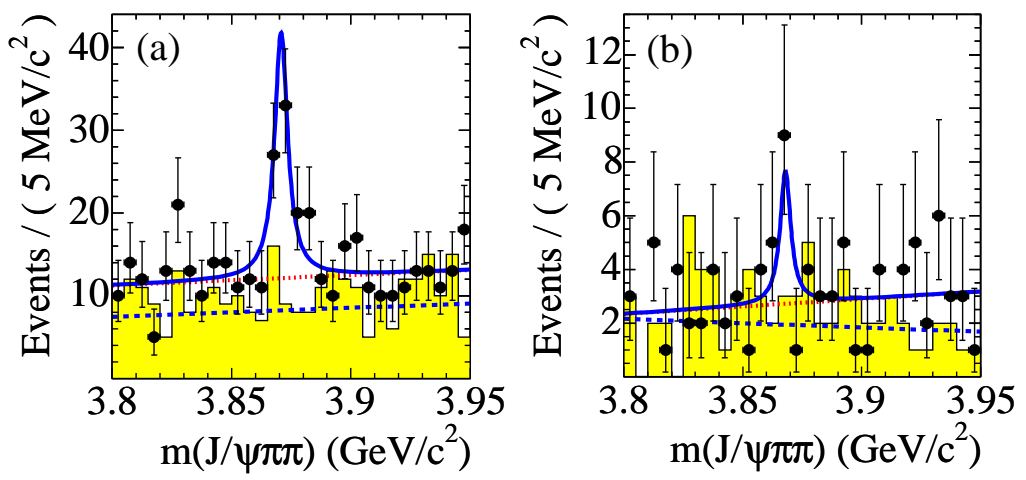

Figure 2. $X(3872)$ state in charged (a) and neutral (b) $B$ decays.

Several other studies have been presented like the inclusive search for $B^{-} \rightarrow X(3872) K^{-}$decays, with a novel technique variant of a recoil technique, $\mathrm{A} B$ is fully reconstructed in one side and a two body decay with a charged kaon is searched on the other side, allowing an absolute BF measurement ${ }^{8}$. Among the other new results we confirmed the evidence for the radiative decay $X(3872) \rightarrow J / \psi \gamma^{9}$. 


\subsection{Studies of the broad $Y(4260)$ structure}

Another puzzling state was discovered last year by BaBar ${ }^{10}$ in ISR events $e^{+} e^{-} \rightarrow \gamma_{I S R} J / \psi \pi^{+} \pi^{-}$decays. A broad structure in $J / \psi \pi^{+} \pi^{-}$mass around $4260 \mathrm{MeV} / \mathrm{c}^{2}$ called $Y(4260)$ was observed with more than $8 \sigma$ significance with an obvious assignment of quantum numbers $J^{P C}=1^{--}$.

Surprisingly this structure is not observed in $e^{+} e^{-} \rightarrow$ hadrons cross section and it seen in this mode even if it is already above $D^{*} D^{*}$ threshold.

Also here there are several interpretations alternative to conventional charmonium. So it is important to search for the Y(4260) with other production mechanisms and in other decay modes. While there are no indication so far in other ISR-produced Final States like $Y(4260) \rightarrow p \bar{p}$, $\phi \pi^{+} \pi^{-}$(excluding glueball hyphothesis), $D \bar{D}^{9}$ (where a large BF would be expected), there is a $3.1 \sigma$ indication of $\mathrm{Y}(4260)$ in $\mathrm{B}$ decays ${ }^{7}$.

\section{Conclusions}

The last years have been very productive for quarkonium spectroscopy studies at the $B$ factories and in particular for BaBar. We have shown the observation for the first time the $\Upsilon(4 S) \rightarrow \pi^{+} \pi^{-} \Upsilon(2 S)$ decay. We have discussed new results on new charmonium-like states, $X(3872)$ and $Y(4260)$, that are searched both in charged and neutral B decays together with a kaon, and in initial state radiation events.

\section{References}

1. Belle Coll., hep-ex/0512034.

2. BaBar Coll., Phys. Rev. Lett. 96, 232001 (2006).

3. See for instance: Kuan-Yan, Phys. Rev. D24 2874 (1981), and many others.

4. For a discussion on charmonium spectroscopy see: T.Barnes, S.Godfrey and E.S.Swanson Phys.Rev. D72 054026 (2005).

5. Belle Coll., Phys. Rev. Lett. 91262001 (2003).

6. CDF Coll., Phys. Rev. Lett. 93072001 (2004); D0 Coll., Phys. Rev. Lett. 93 162002 (2004); BaBar Coll., Phys. Rev. D71 071103 (2005).

7. BaBar Coll., Phys. Rev. D73 011101 (2006).

8. BaBar Coll., Phys. Rev. Lett. 96052002 (2006).

9. BaBar Coll., preliminary result sent to winter 2006 conferences.

10. BaBar Coll., Phys. Rev. Lett. 95142001 (2005). 